\title{
Ultrafast energy transfer between water molecules
}

\author{
T. Jahnke ${ }^{1 \star}$, H. Sann ${ }^{1}$, T. Havermeier ${ }^{1}$, K. Kreidi ${ }^{1}$, C. Stuck$^{1}$, M. Meckel ${ }^{1}$, M. Schöffler ${ }^{2}$, N. Neumann ${ }^{1}$, \\ R. Wallauer ${ }^{1}$, S. Voss ${ }^{1}$, A. Czasch ${ }^{1}$, O. Jagutzki ${ }^{1}$, A. Malakzadeh ${ }^{1}$, F. Afaneh ${ }^{3}$, Th. Weber ${ }^{2}$, \\ H. Schmidt-Böcking ${ }^{1}$ and R. Dörner ${ }^{1}$
}

\begin{abstract}
At the transition from the gas to the liquid phase of water, a wealth of new phenomena emerge, which are absent for isolated $\mathrm{H}_{2} \mathrm{O}$ molecules. Many of those are important for the existence of life, for astrophysics and atmospheric science. In particular, the response to electronic excitation changes completely as more degrees of freedom become available. Here we report the direct observation of an ultrafast transfer of energy across the hydrogen bridge in $\left(\mathrm{H}_{2} \mathrm{O}\right)_{2}$ (a so-called water dimer). This intermolecular coulombic decay leads to an ejection of a low-energy electron from the molecular neighbour of the initially excited molecule. We observe that this decay is faster than the proton transfer that is usually a prominent pathway in the case of electronic excitation of small water clusters and leads to dissociation of the water dimer into two $\mathrm{H}_{2} \mathrm{O}^{+}$ions. As electrons of low energy $(\sim 0.7-20 \mathrm{eV})$ have recently been found to efficiently breakup DNA constituents ${ }^{1,2}$, the observed decay channel might contribute as a source of electrons that can cause radiation damage in biological matter.
\end{abstract}

The water molecule is, as a triatomic molecule, rather simple in structure and its geometry is well known. In contrast to that, the interplay of compounds of water molecules or other atoms and molecules with water, for example in a solution, is very rich and far from being fully understood. At the very onset of condensation when two water molecules are combined to form a water dimer a new dimension of complexity arises: electronic excitation of this complex spawns nuclear dynamics leading to fragmentation into a protonated fragment (that is, $\mathrm{H}_{3} \mathrm{O}^{+}$) and an $\mathrm{OH}$ group ${ }^{3,4}$. For this fragmentation, first a proton migrates from one of the molecules to its neighbour, usually along a distance that is larger than the bond lengths found in the water molecule itself. Such fragmentation dynamics are characteristic for larger clusters, as well ${ }^{5}$. Typical mass spectra of fragments of water droplets show a break-up into protonated cluster fragments $\left(\mathrm{H}_{2} \mathrm{O}\right)_{n} \mathrm{H}^{+}$of different sizes and into $\mathrm{OH}$ groups. A reason for this is the absence of direct transitions within the Franck-Condon region to break-up channels that do not involve proton migration ${ }^{6-8}$. Furthermore, the migration itself is highly efficient and occurs on a timescale of $<60$ fs (ref. 9).

The response of condensed water to electronic excitation has far-reaching consequences for biological systems. Radiation damage to cells naturally depends sensitively on the routes by which energy deposited into the cells is finally distributed and which fragmentation and de-excitation pathways are favoured. Experiments have shown that the constituents of DNA are highly vulnerable to low-energy electrons ${ }^{1}$. These studies revealed that not only does primary ionization by high-energy particles or photons cause damage, but also that low-energy electrons in particular break-up biomolecules efficiently ${ }^{2}$.
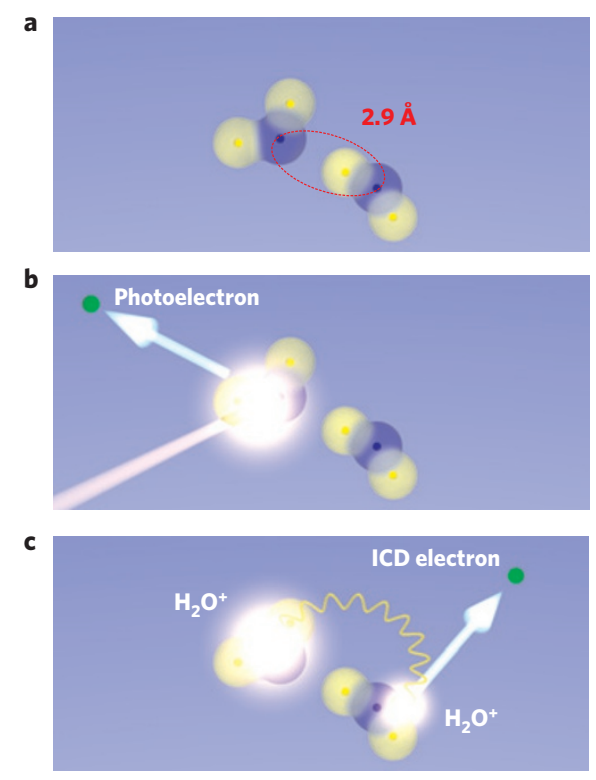

Figure 1 | Investigated species and process. a, Geometry of the water dimer (adapted from ref. 21). The red oval shows an internuclear distance of $2.9 \AA$ with a corresponding KER of $4.9 \mathrm{eV}$ after the photo reaction. b,c, The process observed in this experiment: an electron from the inner valence shell of one of the molecules of the dimer is ejected by absorption of a photon (b) and then the energy released by de-excitation at this site is transferred to the neighbouring site from where a second, low-energy electron is emitted (c)

Here, we report the observation that inner-valence-ionized water dimers fragment, contrary to the standard scenario described above. They relax ultrafast and directly, without a preceding migration of protons. Their de-excitation is observed to occur along with the emission of a low-energy electron that hasdepending on the states involved-an energy less than $10 \mathrm{eV}$. That energy range coincides with the energy range relevant for radiation damage. The relaxation occurs through an intermolecular coulombic decay (ICD), a process first predicted by Cederbaum and co-workers 12 years ago ${ }^{10}$. ICD occurs when the excited particle is only loosely attached to neighbouring particles by for example, Van der Waals forces or hydrogen bonding. In such a scenario, an intermolecular decay involving the emission of an electron from a neighbouring partner of the initially excited particle may become the dominant channel for de-excitation. ICD is a highly efficient ionization mechanism and happens for species investigated so far on timescales less than $100 \mathrm{fs}$. Other intermolecular or interatomic

${ }^{1}$ Institut für Kernphysik, University of Frankfurt, Max-von-Laue-Str. 1, D-60438 Frankfurt, Germany, ${ }^{2}$ Lawrence Berkeley National Laboratory, Berkeley, California 94720, USA, ${ }^{3}$ Physics Department, The Hashemite University, PO Box 150459, Zarqa 13115, Jordan. *e-mail: jahnke@atom.uni-frankfurt.de. 


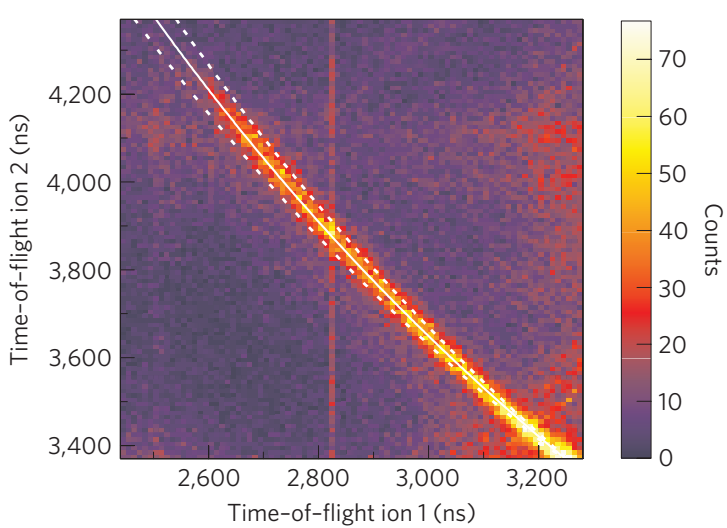

Figure $\mathbf{2}$ | Correlation of the times-of-flight of the two measured ions.

The distribution reveals the decay of the dimer into $\mathrm{H}_{2} \mathrm{O}^{+}+\mathrm{H}_{2} \mathrm{O}^{+}$(solid white line). The $\mathrm{V}$-shaped dashed white line shows the expected position of events for the break-up after proton migration, that is, into $\mathrm{H}_{3} \mathrm{O}^{+}+\mathrm{OH}^{+}$

ionization mechanisms, for example electron impact ionization after photoionization, are typically one order of magnitude less probable. It occurs after ionization of an appropriate atomic shell (that is, an inner valence shell) ${ }^{11-13}$, after excitation ${ }^{14-16}$ and especially as a terminal step after Auger decay ${ }^{17-19}$ and therefore after ionization of the cluster compound with high-energy particles or photons. Most recently the main characteristic of ICD, ionization of nearest-neighbour molecules by energy transfer, was also seen in the core-hole relaxation of $\mathrm{OH}^{-}$dissolved in water ${ }^{20}$. In all cases except the last one, the electron being emitted as a result of ICD is of low energy. As ICD happens at some step of a possible reaction chain, it is nearly independent of the energy of the initially ionizing particle. Therefore, ICD is an efficient and general mechanism for converting high-energy radiation into low-energy free electrons.

Providing experimental evidence that this decay occurs in water clusters is not straightforward, even if it is the dominant decay channel. Its main feature is a low-energy electron being emitted from a different site than the originally excited one. In larger clusters, inelastic electron scattering usually leads to a photoelectron spectrum with kinetic energies down to zero energy masking possible ICD electrons. To avoid this problem, we investigate the water dimer instead of larger droplets. This has the advantage that all charged particles that are created during the process can be measured in coincidence and in addition the system is small enough to allow for theoretical modelling of all steps of the process in the future. Model calculations for the primary ICD step in water dimers for a fixed geometry have already been reported ${ }^{21}$.

We trigger the process by removing an inner-valence electron from one of the water molecules of the dimer. After ICD has occurred, a second electron is emitted and the second water molecule of the dimer becomes charged (see Fig. 1b,c). By measuring the mass, charge, direction and energy of both ions and both electrons, we obtain a complete picture of the reaction. First, we can check for momentum conservation and thus show that the particles originate from the same event of ionization and that the two water ions initially formed a water dimer. From the mass spectrum, we can tag which of the bonds broke and identify potential proton migration. From the energy of the ions, we can deduce the internuclear distance of the two ions at the time the Coulomb explosion was triggered. That internuclear distance can be compared with the values for the ground state of the dimer found in the literature, giving a first hint of the timescale of the decay dynamics and therefore on the efficiency of ICD in competition with other processes. The emitted electrons provide a fingerprint of the decay process: we expect a photoelectron within a certain energy

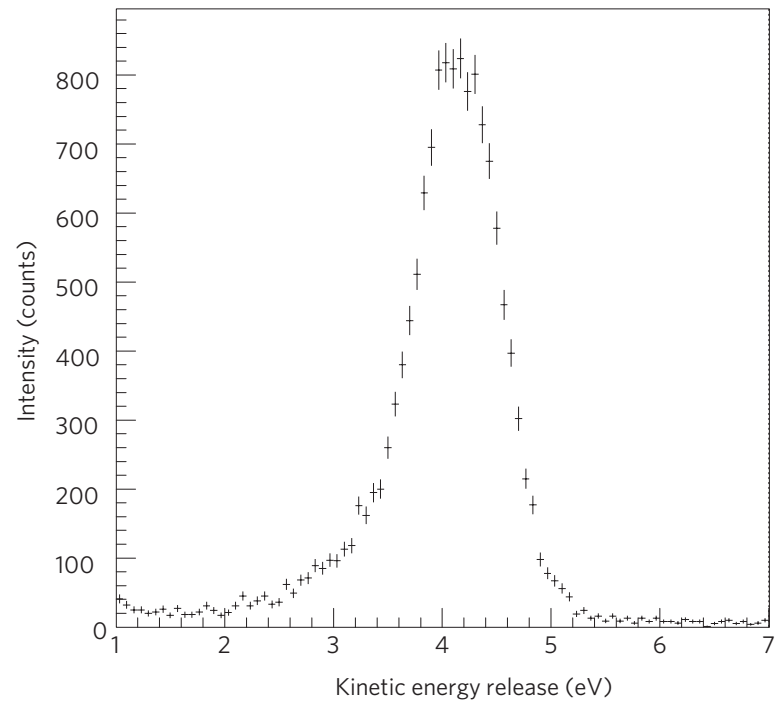

b

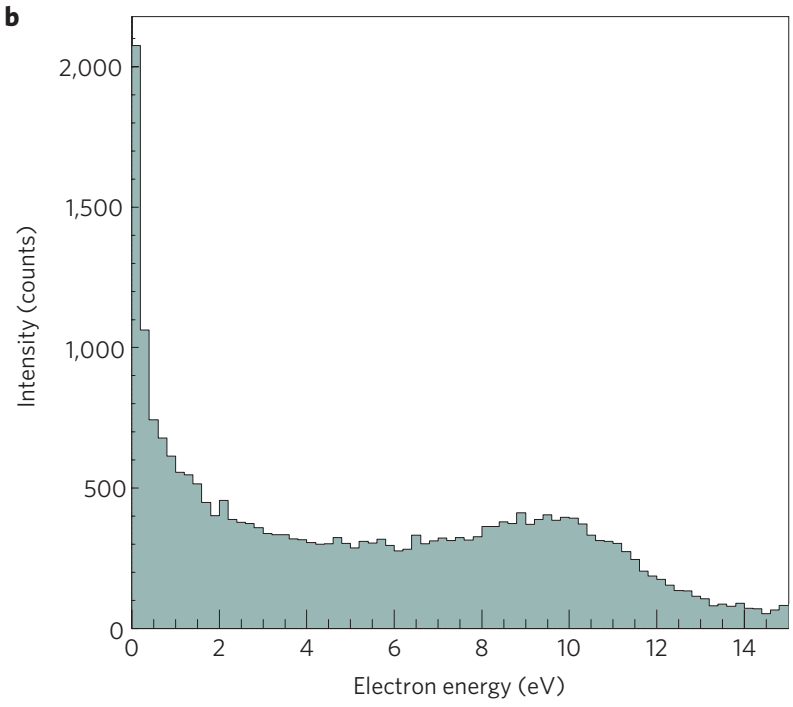

Figure 3 | Energies of the particles measured in the experiment. a, Measured KER of the ions after break-up of the dimer into $\mathrm{H}_{2} \mathrm{O}^{+} / \mathrm{H}_{2} \mathrm{O}^{+}$ An energy of $4.9 \mathrm{eV}$ corresponds to an internuclear distance of $2.9 \AA$ as indicated in Fig. 1a. The error bars correspond to the statistical error. b. Measured electron kinetic energy for break-up of the dimer into $\mathrm{H}_{2} \mathrm{O}^{+} / \mathrm{H}_{2} \mathrm{O}^{+}$.

range, determined by the photon energy and the binding energy of the ionized orbital, and a low-energy ICD electron.

The experiment was carried out at the Berlin synchrotron BESSY II at beamline U125-2/SGM, as described in the Methods section.

During offline analysis, first the break-up channel of the dimer was identified: after emission of two electrons the dimer will fragment into two singly charged ions in a Coulomb explosion. The two ions are emitted back-to-back with momenta of equal magnitude but opposite direction. As the time-of-flight depends on the momentum and the mass of the particle, the back-to-back emission leads to a line structure in the time-correlation map of both particles shown in Fig. 2. Different masses correspond to different lines and an asymmetric break-up into fragments of unequal masses appears as a V-shaped structure. The dashed lines indicate the locus of events for fragmentation after proton migration $\left(\mathrm{H}_{3} \mathrm{O}^{+}+\mathrm{OH}^{+}\right)$and the solid line that of direct fragmentation without proton rearrangement $\left(\mathrm{H}_{2} \mathrm{O}^{+}+\mathrm{H}_{2} \mathrm{O}^{+}\right)$. We do not find indications of proton migration, thus proving that the decay leading to the emission of two electrons is so fast that the 


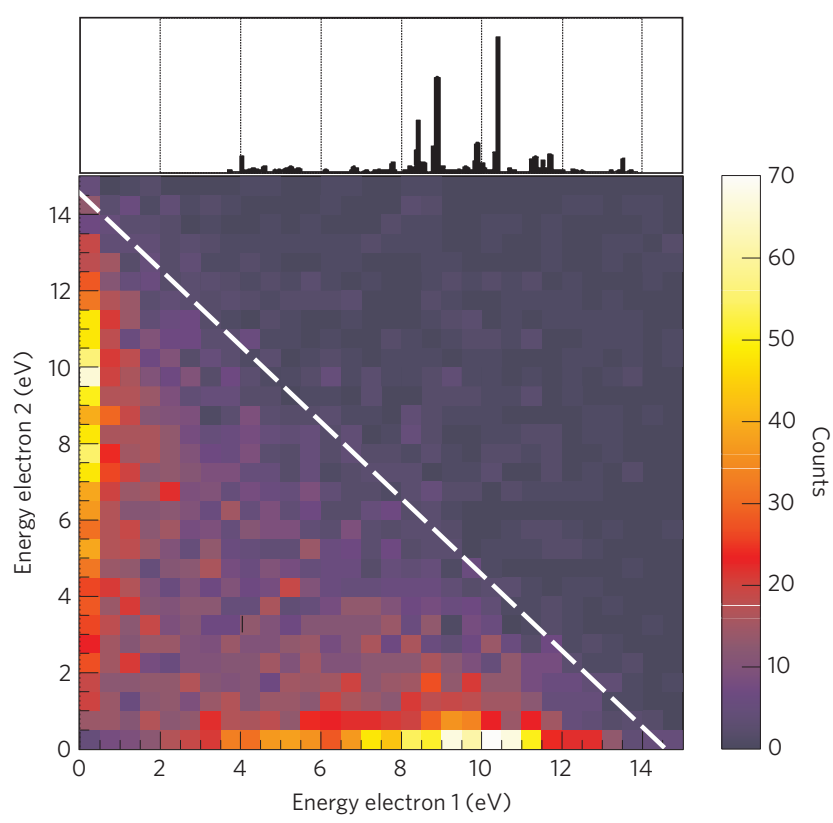

Figure 4 | Energy correlation found for the two electrons measured in coincidence. Bottom: experimental energy distribution of the two electrons measured in coincidence. Top: calculated energies and probabilities for $\left(\mathrm{H}_{2} \mathrm{O}\right)_{2}$ inner-valence photoionization at a photon energy of $43 \mathrm{eV}$, adapted from ref. 21.

water dimer explodes by Coulomb repulsion before there is time for a proton to change its position.

To unveil the mechanisms by which the photon energy dumped at one site is transferred across the dimer, we now investigate the ion and electron energies. Figure $3 \mathrm{a}$ shows the measured sum of the kinetic energies of the two $\mathrm{H}_{2} \mathrm{O}^{+}$ions (kinetic energy release, $\mathrm{KER})$. At large internuclear distances, the intermolecular potential drops with $1 / R$ given by the Coulomb repulsion between the two charged centres at the internuclear distance $R$. Therefore, the intermolecular distance at the instant of ejection of the second electron can be obtained from the measured KER (ref. 26). A KER of $4.9 \mathrm{eV}$ corresponds to $R=2.9 \AA$. This value nicely corresponds to typical distances of the two molecules of the water dimer in its ground state ${ }^{21}$ as shown by the red oval in Fig. 1a. The measured electron energy spectrum (Fig. 3b) clearly shows apart from the photoelectron distribution a great amount of electrons with low energies as expected for the ICD process.

Finally, Fig. 4 shows the energy of the two electrons measured in coincidence. First, it shows energy conservation: for the case of inner-valence ionization and a photon energy of $h v=43 \mathrm{eV}$, the maximum sum energy of the two electrons is approximately $14.5 \mathrm{eV}$ depending on the orbitals involved in the decay and the KER. Apart from that, two sharp features linking an electron of nearly zero energy to a broad distribution of higher energies are visible. A comparison to calculated binding energies (Fig. 4 (top), adapted from ref. 21) shows that the distribution from approximately 4 to $13 \mathrm{eV}$ corresponds to a photoelectron leaving the singly charged water dimer ion in a single inner-valance hole state. These photoelectrons are accompanied by a second, low-energy electron from the ICD. The electrons at zero energy show this most prominently, but as for the manifold of involved states other combinations of photoelectrons and ICD electrons with a sum energy $<14.5 \mathrm{eV}$ occur, as well, just as Fig. 4 reveals. The calculated spectrum in Fig. 4 shows narrow lines for the photoelectron spectrum because it corresponds to a fixed geometry of the dimer, neglecting the decay width and, more importantly, the distribution of angles and internuclear distances in the ground and excited states. Including those would lead to widely overlapping, broadened lines in a measured electron energy spectrum.

Our work unveils the presence of an intermolecular decay in loosely bound matter. Similar findings were recently observed in another experiment on larger water clusters ${ }^{28}$. The elaboration of the role of this mechanism in radiation damage of biological systems in detail is beyond the scope of this experiment. However, that role could be explored in future investigations: with our set-up, any species that can be prepared as a molecular beam can be examined. Experiments similar to those described in ref. 2 are conceivable. Instead of using an electron gun as a donor for electrons, the experiment could be carried out using an aqueous solution of the target species. In that case, the complete pathway of the damage can be examined: from the fragments occurring after the reaction being triggered by photo ionization it can be deduced which electron attached to the DNA constituent. That way the significance of intermolecular decays to radiation damage can be directly investigated and separated from other sources of damage.

\section{Methods}

The experiment was carried out at the Berlin synchrotron BESSY II at beamline U125-2/SGM in a single-bunch operation using cold-target recoil-ion-momentum spectroscopy ${ }^{22-24}$. A photon beam with an energy of $h v=43 \mathrm{eV}$ was crossed with a preheated supersonic jet consisting of water vapour. We achieved a sufficiently dense target with a reasonable fraction of condensed water molecules by heating the nozzle, the gas line and the water reservoir to a temperature of $110^{\circ} \mathrm{C}, 110^{\circ} \mathrm{C}$ and $95^{\circ} \mathrm{C}$ respectively. Under these conditions, the stagnation pressure of the vapour was 0.8 bar, leading to a dimer fraction of less than $1 \%$, whereas the production of larger clusters was still suppressed: a trimer fraction of less than one tenth of the dimers was deduced from mass spectroscopy measurements. A cold-target recoil-ion-momentum spectroscopy analyser consisting of two multi-channel plate detectors with a delay-line position readout ${ }^{25}$ was used to carry out the coincident measurement of all reaction products. In brief, a homogeneous electric field guides electrons and ions to two opposing position- and time-sensitive detectors. By measuring the time-of-flight and the position of impact for each particle their vector momentum after the photo reaction is obtained. The information is recorded as raw timing as a list-mode data set. From that, momenta are calculated in an offline analysis where, furthermore, constraints (for example, by checking for momentum conservation for each photo ionization event recorded) can be applied to the data set, reducing the background. A superimposed homogeneous magnetic field confines the electrons to the spectrometer volume. With values of $E=9.6 \mathrm{~V} \mathrm{~cm}^{-1}$ for the homogeneous electric and $B=7 \mathrm{G}$ for the magnetic extraction field, electrons with an energy up to $12 \mathrm{eV}$ and ions with an energy up to $8 \mathrm{eV}$ can be detected within a $4 \pi$ emission solid angle. The electron arm of the analyser used McLaren-time focusing ${ }^{27}$ and a hexagonal delay-line anode (Roentdek HEX-80, see http://www.roentdek.com for details on the detectors) was used to reduce the dead-time of the electron detector, enabling the coincident measurement of the two electrons.

Received 22 June 2009; accepted 25 November 2009; published online 10 January 2010

\section{References}

1. Boudaiffa, B., Cloutier, P., Hunting, D., Huels, M. A. \& Sanche, L. Resonant formation of DNA strand breaks by low-energy (3-20 eV) electrons. Science 287, 1658-1660 (2000).

2. Hanel, G. et al. Electron attachment to uracil: Effective destruction at subexcitation energies. Phys. Rev. Lett. 90, 188104 (2003).

3. Angle, L. \& Stace, A. J. Dissociation patterns of $\left(\mathrm{H}_{2} \mathrm{O}\right)_{n}^{+}$cluster ions, for $n=26$. Chem. Phys. Lett. 345, 277-281 (2001).

4. Belau, L., Wilson, K. R., Leone, S. R. \& Ahmed, M. Vacuum ultraviolet (VUV) photoionization of small water clusters. J. Phys. Chem. A 111, 10075-10083 (2007).

5. Dong, F., Heinbuch, S., Rocca, J. J. \& Bernstein, E. R. Dynamics and fragmentation of van der Waals clusters: $\left(\mathrm{H}_{2} \mathrm{O}\right)(n),\left(\mathrm{CH}_{3} \mathrm{OH}\right)(n)$, and $\left(\mathrm{NH}_{3}\right)(n)$ upon ionization by a $26.5 \mathrm{eV}$ soft X-ray laser. J. Chem. Phys. 124, 224319 (2006).

6. Shiromaru, H., Shinohara, H., Washida, N., Yoo, H. S. \& Kimura, K. Synchrotron radiation measurements of appearance potentials for $\left(\mathrm{H}_{2} \mathrm{O}\right)_{2}^{+}$, $\left(\mathrm{H}_{2} \mathrm{O}\right)_{3}^{+},\left(\mathrm{H}_{2} \mathrm{O}\right)_{2} \mathrm{H}^{+}$and $\left(\mathrm{H}_{2} \mathrm{O}\right)_{3} \mathrm{H}^{+}$in supersonic jets. Chem. Phys. Lett. 141, 7-11 (1987)

7. Radi, P. P. et al. Femtosecond photoionization of $\left(\mathrm{H}_{2} \mathrm{O}\right)_{n}$ and $\left(\mathrm{D}_{2} \mathrm{O}\right)_{n}$ clusters. J. Chem. Phys. 111, 512-518 (1999).

8. Tachikawa, H. Ionization dynamics of the small-sized water clusters: A direct ab initio trajectory study. J. Phys. Chem. A 108, 7853-7862 (2004). 
9. Furuhama, A., Dupuis, M. \& Hirao, K. Reactions associated with ionization in water: A direct ab initio dynamics study of ionization in $\left(\mathrm{H}_{2} \mathrm{O}\right)_{17}$. J. Chem. Phys. 124, 164310 (2006).

10. Cederbaum, L. S., Zobeley, J. \& Tarantelli, F. Giant intermolecular decay and fragmentation of clusters. Phys. Rev. Lett. 79, 4778-4781 (1997).

11. Marburger, S., Kugeler, O., Hergenhahn, U. \& Möller, T. Experimental evidence for interatomic coulombic decay in Ne clusters. Phys. Rev. Lett. 93, 203401 (2003)

12. Jahnke, T. et al. Experimental observation of interatomic coulombic decay in neon dimers. Phys. Rev. Lett. 93, 163401 (2004).

13. Morishita, Y. et al. Experimental evidence of interatomic coulombic decay from the Auger final states in argon dimers. Phys. Rev. Lett. 96, 243402 (2006).

14. Barth, S. et al. Observation of resonant interatomic coulombic decay in $\mathrm{Ne}$ clusters. J. Chem. Phys. 122, 241102 (2005).

15. Aoto, T. et al. Properties of resonant interatomic coulombic decay in Ne dimers Phys. Rev. Lett. 97, 243401 (2006).

16. Jahnke, T. et al. Experimental separation of virtual photon exchange and electron transfer in interatomic coulombic decay of neon dimers. Phys. Rev. Lett. 99, 153401 (2007)

17. Kreidi, K. et al. Localization of inner shell photo electron emission and ICD in neon dimers. J. Phys. B 41, 101002 (2008).

18. Kreidi, K. et al. Relaxation processes following 1 s photoionization and Auger decay in $\mathrm{Ne}_{2}$. Phys. Rev. A 78, 043422 (2008).

19. Ueda, K. et al. Interatomic coulombic decay following the Auger decay: Experimental evidence in rare-gas dimers. J. Electron Spectrosc. Relat. Phenom. 3-10, 166-167 (2008).

20. Aziz, E. F., Ottosson, N., Faubel, M., Hertel, I. V. \& Winter, B. Interaction between liquid water and hydroxide revealed by core-hole de-excitation. Nature 455, 89-91 (2008).

21. Müller, I. B. \& Cederbaum, L. S. Ionization and double ionization of small water clusters. J. Chem. Phys. 125, 204305 (2006).

22. Dörner, R. et al. Cold target recoil ion momentum spectroscopy: A 'momentum microscope' to view atomic collision dynamics. Phys. Rep. 330, 96-192 (2000).
23. Ullrich, J. et al. Recoil-ion and electron momentum spectroscopy: Reaction-microscopes. Rep. Prog. Phys. 66, 1463-1545 (2003).

24. Jahnke, T. et al. Multicoincidence studies of photo and Auger electrons from fixed-in-space molecules using the COLTRIMS technique. J. Electron Spectrosc. Relat. Phenom. 73, 229-238 (2004).

25. Jagutzki, O. et al. A broad-application microchannel-plate detector system for advanced particle or photon detection tasks: Large area imaging, precise multi-hit timing information and high detection rate. NIM A 477, 244-249 (2002).

26. Gislason, E. A. Series expansion for Franck-Condon factors 1. Linear potential and reflection approximation. J. Chem. Phys. 58, 3702-3707 (1973).

27. Wiley, W. C. \& McLaren, I. H. Time-of-flight mass spectrometer with improved resolution. Rev. Sci. Instrum. 26, 1150-1157 (1955).

28. Mucke, M. et al. A hitherto unrecognized source of low-energy electrons in water. Nature Phys. doi:10.1038/nphys1500 (2010).

\section{Acknowledgements}

We would like to thank the staff at BESSY, especially H. Pfau and G. Reichardt, for extraordinary support during the beamtime. Many discussions on ICD with L. Cederbaum and his group are gratefully acknowledged. This work was supported by the DFG and BESSY GmbH.

\section{Author contributions}

Experiment design and set-up (T.J., T.H., K.K., H.S., O.J.), beamtime (T.J., H.S., T.H., K.K., C.S., M.M., M.S., N.N., R.W., S.V., F.A.), data analysis (T.J.), interpretation of data (T.J., R.D., T.W., A.M., H.S.B.), manuscript preparation (T.J., R.D., T.W., M.S., A.C.).

\section{Additional information}

The authors declare no competing financial interests. Reprints and permissions information is available online at http://npg.nature.com/reprintsandpermissions. Correspondence and requests for materials should be addressed to T.J. 